Western University

Scholarship@Western

Department of Economics Research Reports

Economics Working Papers Archive

1983

\title{
Sampling Distribution of Shrinkage Estimators and Their F-Ratios in the Regression Model
}

Aman Ullah

RAL Carter

Virendra K. Srivastava

Follow this and additional works at: https://ir.lib.uwo.ca/economicsresrpt

Part of the Economics Commons

Citation of this paper:

Ullah, Aman, R AL Carter, Virendra K. Srivastava. "Sampling Distribution of Shrinkage Estimators and Their F-Ratios in the Regression Model." Department of Economics Research Reports, 8324. London, ON: Department of Economics, University of Western Ontario (1983). 


\section{RESEARCH REPORT 8324 \\ SAMPLING DISTRIBUTION OF SHRINKAGE \\ ESTIMATORS AND THEIR F-RATIOS IN THE REGRESSION MODEL}

by

Aman UIlah

Richard A. L. Carter

Virendra K. Srivastava ${ }^{1}$

\section{University of Western Ontario \\ and}

Lucknow Univer sity

September, 1983

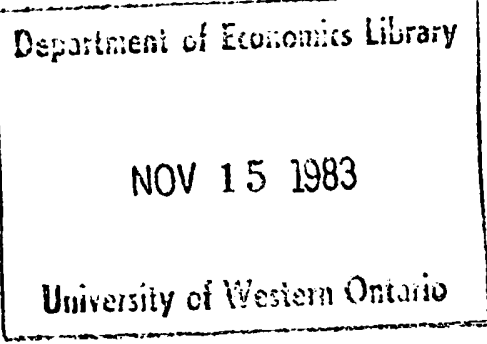




\section{INTRODUCTION}

Several families of adaptive shrinkage estimators have been developed for the estimation of coefficients in a linear regression model, and conditions for their dominance over least squares according to the total mean squared error (risk) criterion have been obtained; see, for example, Judge et al (1980, Ch. 12), Judge and Bock (1978) and Vinod and Ullah (1981, Ch. 5). In a special case of the Stein-type shrinkage estimator, U11ah (1974, 1982) has attempted to analyze its approximate marginal sampling distribution analytically. However, no attempts have been made to evaluate the sampling distribution of other adaptive shrinkage estimators and especially of the $t$ and $F$ ratios which are of significant interest for applied researchers. This paper is an attempt in these directions. We have considered a general family of adaptive shrinkage estimators and have derived Edgeworth-type asymptotic expansions in order to approximate the exact multivariate as well as marginal sampling distributions. The asymptotic expansions of the F ratio are also derived. Although all these expansions are derived by assuming $\sigma$ (standard error of the disturbance) to be smaIl, the asymptotic results for large $\mathrm{T}$ are also given. It is shown that the small- $\sigma$ approximation provides high order of accuracy to the exact sampling distribution of the $F$ ratio compared to the large-T approximation. This is a new result and it is useful for getting the correct size of the test. Further, it suggests the use of the sma11- $\sigma$ technique to derive the approximate distributions of various test statistics in econometrics whose exact sampling distributions are not known. Another interesting result in the paper is the examination 
of the performance of shrinkage estimators with respect to the least squares estimator according to a new criterion; viz., concentration of the distribution around the true value. We find that the dominance condition under the mean squared error criterion is robust against the new criterion.

The plan of the paper is as follows. We present the family of shrinkage estimators in Section 2 and its multivariate as well as marginal distributions in 2.1. In 2.1 we also compare the performance of the shrinkage estimators with the least squares estimator. Then in Section 2.2 we present the sampling distribution of the $F$ ratio. In Section 3 a numerical experiment is conducted to assess the accuracy of the sma11- $\sigma$ approximation compared to the large-T approximation. Finally in section 4 we present the proofs of the theorems given in Section 2.

\section{THE MAIN RESULTS}

Consider a linear regression model:

(2.1) $\quad y=x \beta+u$

where $\mathrm{y}$ is a $\mathrm{T} \times 1$ vector of $\mathrm{T}$ observations on the variables to be explained, $X$ is a $T X P$ full colum rank matrix of $T$ observations on $p$ explanatory variables, $\beta$ is a $p \times 1$ vector of regression coefficients and $u$ is $a \mathrm{~T} \times 1$ vector of disturbances assumed to follow a multivariate normal distribution $N\left(0, \sigma^{2} I_{T}\right)$.

The least squares estimator of $\beta$ is

(2.2) $\quad b=\left(x^{\prime} x\right)^{-1} x^{\prime} y$

which is unbiased and has a multivariate normal distribution $N\left(\beta, \sigma^{2}\left(x^{\prime} x\right)^{-1}\right.$. 
Now consider a general class of shrinkage estimators

$$
\text { (2.3) } \hat{\beta}=[I+h A]^{-1} b \text {, }
$$

where $A$ is any known $p \times p$ positive definite matrix and the stochastic scalar $h$ is

(2.4) $\quad h=\frac{k \hat{u}^{\prime} \hat{u}}{b^{\prime} B b} ; \hat{u}=y-X b=M$ and $M=I-X\left(X^{\prime} X\right)^{-1} X^{\prime}$.

In $(2.4), k$ is a positive constant and $B$ is a known positive definite matrix.

When $A=I_{p}$ and $B=X^{\prime} X, \hat{B}$ becomes the Stein-type Ullah and Ullah (1978) estimator

(2.5) $\hat{\mathrm{b}}=\left[1-\frac{k \hat{\mathrm{u}}^{\prime} \hat{\mathrm{u}}}{\mathrm{b}^{\prime} \mathrm{X}^{\prime} \mathrm{Xb}+k \hat{\mathrm{u}}^{\prime} \hat{\mathrm{u}}}\right] \mathrm{b}$.

Also for $A=\left(X^{\prime} X\right)^{-1}, \hat{B}$ is an adaptive ridge-type estimator. Various other Bayesian and non-Bayesian adaptive shrinkage estimators can also be shown as special cases of (2.3); for details, see Judge and Bock (1978), Judge et al (1980) and Vinod and Ullah (1981, Cb. 13). The mean squared error properties of $\hat{\beta}$ under normal and non-normal disturbances have been analyzed in Ullah et al (1983).

We analyze here the small- $\sigma$ approximation for the distribution of $\hat{B}$ and the $F$ ratio given in $(2.26)$. These results are asymptotic in nature and require $\sigma$ to be sma11; see Kadane (1971). Nagar (1959) type large-T asymptotic approximations are easily derived from the smal1- $\sigma$ asymptotic expressions. The results have been derived here using the small- $\sigma$ approach for three reasons. First, it is simpler to develop the asymptotic expansion of a general class of estimators, like $\hat{\beta}$, using this approach: large-T expansions would require assumptions about the nature of $k$ and the matrices 
$A$ and $B$ as $T$ grows large. Secondly, Srivastava et al (1980) and U1lah (1980) have shown, for different estimators, that the sma11- $\sigma$ approach gives better approximations to the exact moments than does the large-T approach. Section 3 shows that this is also true for the small- $\sigma$ approximation of the exact distribution of a test statistic. Thirdly, we note that while in sma11- $\sigma$ approximations the sample size $T$ is fixed, the large- $T$ approximations make sense only for sufficiently large $T$. It may be more typical for researchers to have small samples of data from populations with small error variances, in which case small- $\sigma$ results are more useful.

2.1 The Approximate Distribution Functions of $\hat{\beta}$ and $\hat{\beta}_{i}$

Before presenting the results we define the estimation error of the vector $\hat{\beta}$ and its $i^{\text {th }}$ element $\hat{\beta}_{i}$, respectively, as

$$
\text { (2.6) } \quad x=\frac{1}{\sigma}(\hat{\beta}-\beta) \text { and } r^{\prime} x=\frac{1}{\sigma} r^{\prime}(\hat{\beta}-\beta)=\frac{1}{\sigma}\left(\hat{\beta}_{i}-\beta_{i}\right)
$$

where 2 is $\mathrm{a} p \times 1$ vector with 1 at the $i^{\text {th }}$ place and 0 elsewhere. We also introduce the following notations:

$$
\theta=\beta^{\prime} B \beta, \quad q^{2}=\tau^{\prime} Q \tau, \quad Q=\left(X^{\prime} X\right)^{-1}
$$

$$
\mathrm{n}=\mathrm{T}-\mathrm{p}, \quad \mathrm{m}_{*}=\mathrm{Q}^{-\frac{1}{2}} \mathrm{~m}_{\mathrm{o}}
$$

where $\mathrm{m}_{0}$ is a $\mathrm{p} \times 1$ vector of fixed constants.

THEOREM 1: The sma11- $\sigma$ asymptotic expansion (for fixed $T$ ) of the multivariate distribution function of $|x|$, up to order $0\left(\sigma^{2}\right)$, is

(2.8) $P\left[|x| \leq m_{0}\right]=G\left(m_{*}\right)-G\left(-m_{*}\right)+\frac{\sigma^{2} k n}{\theta^{2}}\left[G\left(m_{*}\right)-G\left(-m_{*}\right)\right] \operatorname{tr}\left(C Q^{\frac{1}{2}} \Gamma Q^{\frac{1}{2}}\right)$

where $G\left(m_{*}\right)$ represents the standard multivariate normal distribution function, $\Gamma$ is a $p \times p$ diagonal matrix with $j^{\text {th }}(j=1, \ldots, p)$ diagonal element as $2 m_{j *} g\left(m_{j *}\right) /\left(G\left(m_{j *}\right)-G\left(-m_{j *}\right)\right) ; g\left(m_{j *}\right)$ represents the standard univariate normal density function, and 
$(2.9)$

$$
C=\theta Q^{-1} A-2 Q^{-1} A B \beta^{\prime} B-\frac{1}{2}(n+2) k Q^{-1} A B \beta^{\prime} A Q^{-1}
$$

Proof: The proof is given in section 4 .

Remark: The $|\mathrm{x}| \leq \mathrm{m}_{\mathrm{o}}$ in $(2.8)$ is equivalent to $-\mathrm{m}_{\mathrm{o}}<\mathrm{x} \leq \mathrm{m}_{\mathrm{o}}$. Thus $P\left[|x| \leq m_{0}\right]=P\left[-m_{0}<x \leq m_{0}\right]$. The statement of the theorem in this form is useful for deriving the result in $(2.13) .^{2}$ Also, the term $\left(G\left(m_{*}\right)-G\left(-m_{*}\right)\right)$ on the righthand side of $(2.8)$ is $\mathrm{P}\left[-\mathrm{m}_{*}<(b-\beta) / \sigma \leq \mathrm{m}_{*}\right]$; that is, it is the probability for the least squares case. The second term is, to order $0\left(\sigma^{2}\right)$, the extent to which the probability of $\hat{\beta}$ is pulled away from that of $b$.

A comparison of $b$ and $\hat{\beta}$ can be made with respect to the concentration of probability around $\beta$. For this purpose we consider the standardized multivariate distribution functions of $b$ and $\hat{\beta}$ and assume for the sake of simplicity that all the elements of $m_{0}$ are identical to a scalar $m$.

It is easy to see that

$$
P\left[Q^{-\frac{1}{2}} \frac{(b-\beta)}{\sigma} \leq m\right]=G(m)
$$

and so $P\left[-m<Q^{-\frac{1}{2}}(b-\beta) / \sigma \leq m\right]=G(m)-G(-m)$, where $G(m)$ is the standard multivariate normal distribution function. Similarly, from (2.8) and (2.9)

$$
\text { (2.11) } \begin{aligned}
P\left[-\mathrm{m}<\mathrm{Q}^{-\frac{1}{2}} \frac{(\hat{\beta}-\beta)}{\sigma} \leq \mathrm{m}\right]=\mathrm{G}(\mathrm{m})-\mathrm{G}(-\mathrm{m}) & +\frac{\sigma^{2} \mathrm{kn \nu}}{\theta^{2}}\left[\theta \operatorname{trA}-2 \beta^{\prime} \mathrm{BA} \beta-\frac{1}{2}(\mathrm{n}+2) \mathrm{k} \beta^{\prime} A Q^{-1} A \beta\right] \\
& \times(G(\mathrm{~m})-G(-\mathrm{m}))
\end{aligned}
$$

where

$$
\text { (2.12) } \nu=\frac{2 m g(m)}{G(m)-G(-m)} \text {. }
$$

We can now present the following result.

COROLIARY 1: Under the criterion of concentration of probability around $\beta$ the shrinkage estimators $\hat{\beta}$ dominates the least squares estimator $b$, that is, 
(2.13) $\quad P\left[-m<\frac{Q}{\sigma}^{-\frac{1}{2}}(\hat{\beta}-\beta) \leq m\right]-P\left[-m<\frac{Q}{\sigma}^{-\frac{1}{2}}(b-\beta) \leq m\right]>0$,

when

(2.14) $0<k<\frac{2}{n+2} \lambda_{\min } \mu_{\max }(d-2), d=\frac{1}{\mu_{\max }} \operatorname{tr} A>2$,

where $\lambda_{\min }$ and $\mu_{\max }$ are the minimum and maximum characteristic roots of

$B\left(A X^{\prime} X A\right)^{-1}$ and $A$, respectively.

Proof: Using (2.10) and (2.11), and noting from Rao (1973, p. 74) that for any two matrices $A$ and $B, \min \left(\beta^{\prime} A B / \beta\right.$ ' $\left.B \beta\right)$. is the minimum root of $|A-\lambda B|=0$, the result in Corollary 1 follows. $\quad$ Q.E.D.

The result in (2.14) provides the dominance condition for the shrinkage estimators $\hat{\beta}$ under a new criterion. It is interesting to observe that this condition of dominance is the same as the dominance condition in v1lah et al (1983, p. 394) under the weighted mean squared error criterion $\mathbf{E}(\hat{\beta}-\beta)^{\prime} Q^{-1}(\hat{\beta}-\beta)-E(b-\beta)^{\prime} Q^{-1}(b-\beta)$ where $Q^{-1}=X^{\prime} X$. Note that the condition (2.14) is independent of the parameters of the model.

In the special case of the Stein-type estimator $\hat{b}$ in (2.5) for which $A=I_{p}$ and $B=X^{\prime} X$ the condition in (2.14) reduces to (2.15) $\quad 0<k<\frac{2}{n+2}(p-2), p>2$.

The results for other special cases of shrinkage estimators can be written similarly with appropriate substitutions of A and B.

Now we consider the distribution of individual elements of $\hat{\beta}$.

THEOREM 2: The small- $\sigma$ asymptotic expansion (for fixed $T$ ) of the distribition function of $\tau^{\prime} x$, up to order $0\left(\sigma^{2}\right)$, is 
(2.16)

$$
P\left[z^{\prime} x \leq m\right]=G\left(\frac{m}{q}\right)+\left(\xi_{0}+\frac{m}{q} \xi_{1}\right) g\left(\frac{m}{q}\right)
$$

where $g(m / q)$ and $G(m / q)$ represent the standard univariate normal density function and distribution function, respectively, m is a scalar constant,

$$
\xi_{0}=\frac{\sigma k n}{\theta q} \imath^{\prime} A \beta, \quad \xi_{1}=-\frac{\sigma^{2} k n}{\theta} \frac{q_{1}}{q^{2}}
$$

and

$$
q_{1}=\tau^{\prime}\left[\frac{1}{2 \theta}\left\{k(n+2) A B \beta^{\prime} A+4 A B B^{\prime} B Q\right\}-A Q\right] \tau
$$

Proof: The proof is given in Section 4.

Remark: An alternative representation of the distribution function of $\tau^{\prime} x=z^{\prime}(\hat{\beta}-\beta) / \sigma$ which is correct up to the order of approximation considered above is

$$
P\left[\tau^{\prime} x \leq m\right]=G\left(\xi_{0}+\frac{m}{q}\left(\xi_{1}+1\right)\right)
$$

This expression can be evaluated by using the standard normal table. ${ }^{3}$ For the special cases of $\hat{\beta}$, the distribution of $\tau^{\prime} x$ can be written immediately from (2.16). We also note that the $P\left[-m<\tau^{\prime} x \leq m\right]=P\left[\tau^{\prime} x \leq m\right]-P\left[\tau^{\prime} x<-m\right]$ coincides with the result in $(2.8)$ when $p=1$.

COROLIARY 2: Under the criterion of concentration of probability around $\tau^{\prime} \beta$ the shrinkage estimators $\tau^{\prime} \hat{\beta}$ dominates the least squares estimator 2'b, that is,

(2.20) $\quad \mathrm{P}\left[-\mathrm{m}<\frac{z^{\prime}(\hat{\beta}-\beta)}{\sigma}<\mathrm{m}\right]-\mathrm{P}\left[-\mathrm{m}<\frac{z^{\prime}(\mathrm{b}-\beta)}{\sigma}<\mathrm{m}\right]>0$,

when

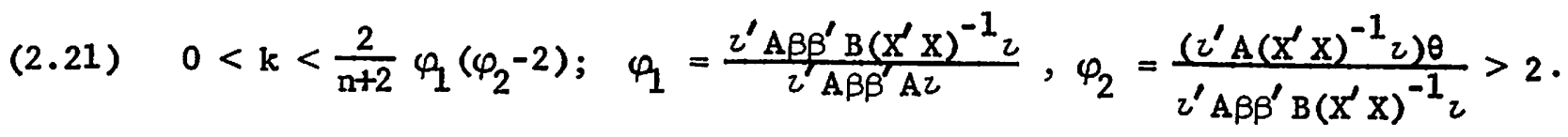

Proof: From (2.16) we note that

$$
\mathrm{p}\left[-\mathrm{m}<\frac{z^{\prime}(\hat{\beta}-\beta)}{\sigma}<\mathrm{m}\right]-\mathrm{P}\left[-\mathrm{m}<\frac{z^{\prime}(\mathrm{b}-\beta)}{\sigma}<\mathrm{m}\right]=2 \frac{\mathrm{m}}{\mathrm{q}} \xi_{1} \mathrm{~g}\left(\frac{\mathrm{m}}{\mathrm{q}}\right) \text {, }
$$


where $\xi_{1}$ is given in $(2.17)$. The result in $(2.21)$ then follows by observing the condition under which $\xi_{1}$ is positive. Q.E.D.

The condition in (2.21) implies that, under the criterion considered, the single element of the shrinkage estimator $\hat{\beta}$ cannot dominate the single element of the least squares estimator $b$ in the entire parameter space. When the matrices $A$ and $B$ are such that $A=\left(X^{\prime} X\right)^{-1} B=A^{\prime}$, the condition in $(2.21)$ reduces to

(2.22) $0<\mathrm{k}<\frac{2}{\mathrm{n}+2}\left(\varphi_{2}-2\right) ; \quad \varphi_{2}=\frac{\left(z_{0}^{\prime} B{ }_{0}\right)\left(\beta^{\prime} B \beta\right)}{\left(z_{0}^{\prime} B \beta\right)^{2}}>2, \tau_{0}=\left(X^{\prime} X\right)^{-1} z_{\text {. }}$

We note, using the Cauchy-Schwartz inequality [Rao (1973), p. 54], that $\varphi_{2} \geq 1$. This implies that for some part of the parameter space $\beta$ the condition $\varphi_{2}>2$ can be satisfied.

For the special cases of $\hat{\beta}$ the conditions of dominance follow by appropriate substitution of $A$ and $B$. For example, in the case of the Steintype estimator $\hat{b}$ in (2.5) we substitute $B=X^{\prime} X$ in (2.22).

To obtain the large-T approximation we first consider $k$ to be any arbitrary constant such that the $\lim (T \mathrm{k})$ is a constant; thus $k$ is of order $T \rightarrow \infty$

$O\left(T^{-1}\right)$. Next we observe that for the different cases of $\hat{\beta}$ the orders of the matrices $A$ and $B$ are different. For example, for the Lawless and Wang (1976) adaptive ridge estimator $A=\left(X^{\prime} X\right)^{-1}$ and $B=X^{\prime} X$; that is, the orders of $A$ and $B$ are $O\left(T^{-1}\right)$ and $O(T)$, respectively. Similarly, for the Steintype estimator $\hat{b}$ in (2.5) the orders of $A=I_{p}$ and $B=X^{\prime} X$ are $O\left(T^{\circ}\right)$ and $O(T)$, respectively. Below we present the result for $\hat{b}$. The distribution of various other shrinkage estimators can be written similarly by appropriate substitutes of the matrices $A$ and $B$. 
COROLIARY 3: The large-T asymptotic expansion of the distribution function of $\tau^{\prime}(\hat{b}-\beta) / \sigma$, up to order $O\left(\mathrm{~T}^{-1}\right)$, is given by

(2.23) $\quad P\left[\frac{\sqrt{\mathrm{T}} t^{\prime}(\hat{b}-\beta)}{\sigma} \leq \mathrm{m}\right]=G\left(\frac{\mathrm{m}}{\sqrt{\mathrm{T} q}}\right)+\left(\xi_{0}^{*}+\frac{m}{\sqrt{\mathrm{T} q}} \xi_{1}^{*}\right) g\left(\frac{m}{\sqrt{\mathrm{T} q}}\right)$

where $\xi_{0}^{*}=\tau^{\prime} \beta(\sigma \mathrm{kT} / \theta \mathrm{q})$ is of order $0\left(\mathrm{~T}^{-\frac{1}{2}}\right)$ and

(2.24) $\quad \xi_{1}^{*}=-\frac{\sigma^{2} k T}{\theta q^{2}}\left[\frac{(k T+4)\left(z^{\prime} \beta\right)^{2}}{2 \theta}-z^{\prime}\left(X^{\prime} X\right)^{-1} z\right]$

is of order $O\left(\mathrm{~T}^{-1}\right)$.

Proof: Noting that $k$ is of order $O\left(T^{-1}\right)$, and $A=I$ and $B=X^{\prime} X$ are of orders $O\left(T^{\circ}\right)$ and $O(T)$, respectively, the result in (2.23) follows. ${ }^{4}$ Q.E.D.

\subsection{Approximate Nu11 Distributions of F Ratio}

Consider the problem of testing $H_{0}: \beta=\beta_{0}$ against $H_{1}: \beta \neq \beta_{0}$ in

model (2.1). The least squares test statistic for this hypothesis is 5

$$
F=\frac{n}{p} \frac{(b-\beta)^{\prime} x^{\prime} \mathbf{x}(b-\beta)}{\hat{u}^{\prime} \hat{u}}=F_{p, n}
$$

The $F$ in (2.25) follows the central $F$ distribution with $p$ and $n$ degrees of freedom under the null hypothesis. Note that when $p=1$ the $F$ becomes the square of a t-ratio.

The improved $F$ is obtained by using $\hat{\beta}$ in place of $b$ in $(2.25)$; that is ${ }^{6}$

$$
F_{\hat{\beta}}=\frac{n}{p} \frac{(\hat{\beta}-\beta)^{\prime} X^{\prime} X(\hat{\beta}-\beta)}{\hat{u}^{\prime} \hat{u}}
$$

In what follows we present the sma11- $\sigma$ and large- $T$ asymptotic expansions of $F_{\beta}^{\wedge}$.

THEOREM 3: The smal1- $\sigma$ asymptotic expansion (for fixed $T$ ) of the distribution of $F \hat{\beta}$, up to order $0\left(\sigma^{2}\right)$, is given by

(2.27) $\quad \mathrm{P}\left[\mathrm{pF}_{\hat{\beta}} \leq \mathrm{m}\right]=\mathrm{G}\left(\mathrm{p} \mathrm{F}_{\mathrm{p}, \mathrm{n}}\right)+\sum_{\ell=1}^{1} \sum_{\ell^{\prime}=1}^{4} \eta_{\ell, \ell^{\prime}} \mathrm{G}\left(\frac{\mathrm{n}(\mathrm{p}+2 \ell)}{\mathrm{n}+2 l^{\prime}} \mathrm{F}_{\mathrm{p}+2 \ell, \mathrm{n}+2 \ell^{\prime}}\right)$ 
where $G\left(\frac{\mathrm{mr}}{\mathrm{w}} F_{r, w}\right)=P\left[\frac{\mathrm{nr}}{\mathrm{w}} F_{r, w} \leq \mathrm{m}\right]$, for any $r$ and $w$, represents the distribution of centra1 $F$ with the $r$ and $w$ degrees of freedom, and $\eta_{-1,1}=\eta_{0,1}=\eta_{-1,2}=$ $=\eta_{1,3}=\eta_{0,4}=\eta_{1,4}=0$, .

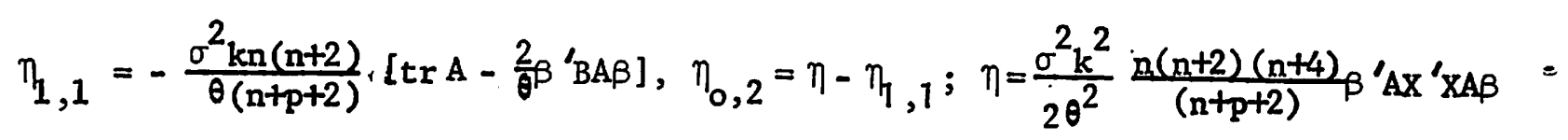

$$
\eta_{1,2}=-\frac{\eta(n+2)}{(n+p+4)}, \eta_{-1,3}=-\eta, \eta_{0,3}=\frac{2 \eta(n+4)}{(n+p+4)}, \eta_{-1,4}=\frac{-\eta(n+6)}{(n+p+4)}
$$

such that $\sum_{\ell=-1}^{1} \sum_{\ell^{\prime}=1}^{4} \eta_{\ell, \ell^{\prime}}=0$.

Proof: The proof is given in Section 4.

The results.for the special cases of shrinkage estimators $\hat{\beta}$ can be written from (2.27) by appropriate substitutions of the matrices A and B. Also, the large-T asymptotic expansions can be obtained easily. We present below the results for Stein-type estimator $\hat{b}$ in (2.5) for which $A=I_{p}$ and $B=X^{\prime} X$. These results have been numerically evaluated in section 3 .

COROLIARY 4: The sma11- $\sigma$ asymptotic expansion, up to order $0\left(\sigma^{2}\right)$, and the large-T asymptotic expansion, up to order $O\left(T^{-1}\right)$, of the distribution of $F_{\hat{b}}$, are respectively given by

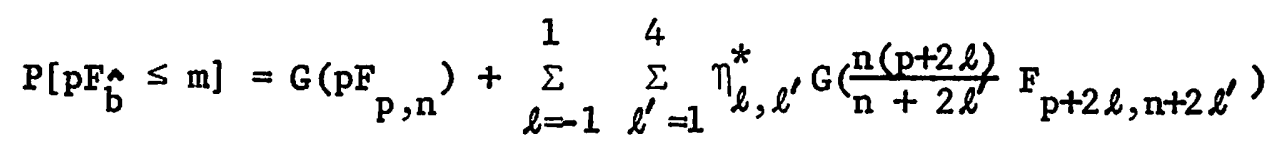

and

(2.30) $\quad \mathrm{P}\left[\mathrm{PF}_{\hat{\mathrm{b}}} \leq \mathrm{m}\right]=\mathrm{G}\left(\mathrm{x}_{\mathrm{p}}^{2}\right)+\sum_{\ell=-1}^{1} \mu_{\ell} \mathrm{G}\left(\mathrm{X}_{\mathrm{p}+2 \ell}^{2}\right)$

where $\eta_{\ell, \ell^{\prime}}^{*}$ is $\eta_{\ell, \ell^{\prime}}$ in $(2.28)$ with $A=I$ and $B=X^{\prime} X, G\left(x_{r}^{2}\right)=P\left[x_{r}^{2} \leq m\right]$ is the distribution of central $x^{2}$ with $r$ degrees of freedom, and

$$
\mu_{0}=\frac{\sigma^{2} k T}{\theta}\left[p-2+\frac{3}{2} k T\right], \mu_{-1}=-\frac{\sigma^{2} k^{2} T^{2}}{\theta}, \mu_{1}=-\frac{\sigma^{2} k T}{\theta}\left[p-2+\frac{1}{2} k T\right]
$$

such that $\sum_{\ell=1}^{1} H_{\ell}=0$. 
Proof: The result in (2.29) follows by direct substitution of $A=I$ and $B=X^{\prime} X$ in (2.27) and (2.28). Further, the result in (2.30) is obtained by observing that $A=0\left(T^{\circ}\right)$ and $B=O(T)$, and noting the fact that the distribution of $(p+2 l) \mathrm{F}_{\mathrm{p}+2 \ell, \mathrm{n}+2 l^{\prime}}$ tends to $x_{\mathrm{p}+2 l}^{2}$ as $\mathrm{T} \rightarrow \infty$.

Remark: We observe from $(2.29)$ (also $(2.27)$ ) that while the sma11- $\sigma$ expansion of the distribution of $F_{\hat{b}}$ is the weighted sum of the central $F$ distributions, from $(2.30)$ the large-T expansion is the weighted sum of central $\chi^{2}$

distributions. ${ }^{7}$ The sum of the weights in each case is unity. The numerical experiment in the following section shows that the small- $\sigma$ exparsion provides high order accuracy compared to large-T expansion for approximating the exact distribution of $\mathrm{F}_{\hat{b}}$. This result suggests the use of small- $\sigma$ approximation in terms of central $F$ distributions for the various other test statistics used in econometrics whose exact sampling distributions are not known.

\section{NUMERICAL RESULTS}

Of all the expansions derived above the most useful for applied work are those dealing with the F ratio; equations $(2.27),(2.29)$ and $(2.30)$, since they are what would be used to build confidence intervals for, or test hypotheses about, linear combinations of regression coefficients. The aim of this section is to use numerical methods to compare the accuracy of the small- $\sigma$ and large-T approximations to the exact distribution function (df) for these improved $F$ ratios. Since this exact df is unknown, the approximate df's are numerically compared to an empirical df obtained by Monte Carlo simulation. 
For the model given in (2.1), assume that the focus of interest is $H_{0}: \beta=\beta_{0}$. The least square test statistic for this hypothesis is as given in (2.25). The improved $F$ ratio is obtained by using $\hat{\beta}$ in place of $b$ and it is as given in (2.26).

In this section the version of $\hat{\beta}$ considered is $\hat{b}$, given by (2.5). This is a special case of the double k-class estimator of Ullah and Ullah (1978).

$$
\hat{b}\left(k_{1}, k_{2}\right)=\left[1-\frac{k_{1} \hat{u}^{\prime} \hat{u}}{y^{\prime} y-k_{2} \hat{u}^{\prime} \hat{u}}\right] b,
$$

obtained by setting $k_{1}=k$ and $k_{2}=1-k_{1}$. Another member of this class is the James-Stein (1961) estimator for which $k_{2}=1.0$. The disadvantage of the James-Stein estimator is that it can have the opposite sign to the least squares estimator. This change of sign can be prevented by choosing $k_{2}$ so that the shrinkage factor $1-\frac{k_{1} \hat{u}^{\prime} \hat{u}}{y^{\prime} y-k_{2} \hat{u}^{\prime} \hat{u}}$ is positive. It is easily shown that a sufficient condition for this is $k_{2} \leq 1-k_{1}$. Note that $\hat{b}$ in $(2.5)$ satisfies this sufficient condition but the James-Stein estimator does not. We prefer this solution to the sign change problem, over those suggested by Baranchik (1964) and Stein (1966), on the grounds that its constant form over repeated samples makes its small sample properties easier to discover .

Since the ratio $F_{\hat{A}}$ will be used to form a confidence region for $\beta$, $\beta$ we wish to set the value of $k_{1}=k$ so as to minimize the expectation of $(\hat{\beta}-\beta){ }^{\prime} X ' X(\hat{\beta}-\beta)$ and hence minimize the radius of the confidence region. The results of UIlah and Ullah can be used to show that, to order $\sigma^{4}$, the value which does this is $k_{1}=k=(p-2) /(n+2)$ : See also Corollary 1 to Theorem 1 above.

In order to numerically evaluate the small- $\sigma$ and large-T approximations, as well as to do the simulation, the value of $T$ was set to 36 and $p$ was 
set to 10 , implying $\mathrm{n}=26$ and $\mathrm{k}=\mathrm{k}_{1}=.2857$. The approximations also involve the ratio of two population parameters

(3.1) $\gamma_{0}=\frac{\sigma^{2}}{\theta}=\frac{\sigma^{2}}{\beta^{\prime} X^{\prime} X \beta}$

Now if we define the population goodness-of-fit to be

$$
\rho^{2}=\frac{\beta^{\prime} X^{\prime} X \beta}{\beta^{\prime} X^{\prime} X \beta+T \sigma^{2}} \text {, }
$$

then we can write this ratio in terms of $\rho^{2}$ as

(3.3) $\quad \gamma_{0}=\frac{1-\rho^{2}}{T}$

This is more convenient than the original form because one typically has a more intuitive understanding of what are "large" or "smal1" values of $\rho^{2}$ than of $\sigma^{2}$ and because this form involves only one unknown parameter instead of two. For the computations discussed here $\rho^{2}$ was set to .70 so that $\gamma_{0}=.0083$

The $\mathrm{X}$ matrix used in the Monte Carlo simulation was derived from a set of 36 observations on 10 regressors which was originally published by Daniel and Wood (1971) and has been used in simulations by Hoerl, Kennard and Baldwin (1975) and Lawless and Wang (1976). Each regressor was first standardized to have zero mean and unit variance so that the 10 slope coefficients could be estimated independently of the intercept. Finally the set of regressors was transformed so that $X$ ' $X$ was a diagonal matrix with the following eigenvalues on the main diagonal: $.07402, .1479, .2318$, $.3605, .6571, .9446,1.051,1.300,1.550,3.686$. This transformation simplifies computation but has no effect on $F$ or $F_{\hat{\beta}}$ or any aspect of their sampling distributions. The ratio of the largest eigenvalue to the smallest is 49.8 which indicates a nontrivial degree of multicollinearity among the regressors. 
The ten items in the vector $\beta$, after the diagonalizing transformation, were each set equal to 1.0. This implies that $\sigma^{2}=\gamma_{0} \beta^{\prime} X^{\prime} X \beta=.119$.

For these parameter values the expectation of the numerator of $F$ is

$$
E\left[(b-\beta)^{\prime} x^{\prime} X(b-\beta)\right]=\sigma^{2} p=1.19
$$

The results of Carter (1981) can be used to find the expectation of the numerator of $\mathrm{F}_{\hat{\beta}}$, to order $\sigma^{8}$,

$$
E\left[(\hat{\beta}-\beta)^{\prime} X^{\prime} X(\hat{\beta}-\beta)\right]=1.11
$$

The generation of random disturbances proceeded in several stages. First the IMSL (1979) subroutine GGUW was used to generate a pair of pseudorandom numbers, $\varepsilon_{1}$ and $\varepsilon_{2}$, from a uniform $(0,1)$ distribution. If $\varepsilon_{1}$ and $\varepsilon_{2}$ are such that $\left(2 \epsilon_{1}-1\right)^{2}+\left(2 \varepsilon_{2}-1\right)^{2} \geq 1.0$ they are rejected and another pair drawn. If $\epsilon_{1}$ and $\epsilon_{2}$ pass this test they are used to construct

$$
\zeta=\left[\frac{-2 \ln \left[\left(2 \varepsilon_{1}-1\right)^{2}+\left(2 \varepsilon_{2}-1\right)^{2}\right]}{\left(2 \varepsilon_{1}-1\right)^{2}+\left(2 \varepsilon_{2}-1\right)^{2}}\right]^{\frac{1}{2}}
$$

which is then used to find

$$
\begin{array}{ll}
(3.7) & \tau_{t}=\zeta\left(2 \epsilon_{1}-1\right)^{2} \\
(3.8) & \tau_{t+1}=\zeta\left(2 \epsilon_{2}-1\right)^{2} .
\end{array}
$$

The pair $\tau_{t}$ and $T_{t+1}$ are pseudo-random drawings from $N(0,1)$. This was repeated for $t=1,3,5, \ldots, 35$ to obtain a vector of $\tau$ of 36 pseudo-random drawings from $N(0,1)$. Then $u=\sigma T$ and a sample $y$, of length 36 , is obtained from $y=x \beta+u$. The procedure was repeated, using the same $x$, to obtain 2500 samples, each of size 36 , which led to 2500 values of $F$ and $F_{\hat{A}}$. The cumulative frequency distribution was obtained for both $F$ and $F_{\hat{B}}$ at intervals of $1 \%$. A few selected points from these cumulative distributions are given in Table 3.1 together with points from the df for the F distribution with 10 and 26 degrees of freedom. 
TABLE 3.1

Selected Points for Cumulative Distributions

\begin{tabular}{cccccc}
$\begin{array}{l}\text { Cumulative } \\
\text { Frequency or } \\
\text { Probability }\end{array}$ & $F_{10,26}$ & F & $\begin{array}{l}\text { Estimated } \\
\text { Sampling } \\
\text { Error }\end{array}$ & $\begin{array}{c}\text { Raw } \\
F_{\hat{\beta}}\end{array}$ & $\hat{F}_{\hat{\beta}}$ \\
\hline .50 & .958721 & .935374 & -.023347 & .874836 & .898183 \\
.75 & 1.365645 & 1.355979 & -.009666 & 1.264360 & 1.274026 \\
.90 & 1.855028 & 1.863848 & -.008820 & 1.736350 & 1.727530 \\
.95 & 2.219718 & 2.258913 & -.039195 & 2.070771 & 2.031576 \\
.99 & 3.094108 & 3.180216 & .086108 & 2.987363 & 2.901255
\end{tabular}

TABLE 3.2

Right Tail Points: Approximate and Simulated Distributions

Cumulative Probability

\begin{tabular}{lcccc} 
& & & & \\
\cline { 2 - 5 } F-value & Simulated & Small- $\sigma$ & Large-T & $F_{10,26}$ \\
\hline 1.7275 & .9000 & .9024 & .9753 & .8726 \\
1.7863 & .9100 & .9138 & .9823 & .8861 \\
1.8462 & .9200 & .9239 & .9879 & .8983 \\
1.9155 & .9300 & .9341 & .9927 & .9109 \\
1.9664 & .9400 & .9407 & .9953 & .9191 \\
2.0316 & .9500 & .9482 & .9978 & .9285 \\
2.1753 & .9600 & .9614 & 1.0001 & .9456 \\
2.3418 & .9700 & .9724 & 1.002 & .9603 \\
2.5521 & .9800 & .9818 & 1.002 & .9732 \\
2.9013 & .9900 & .9907 & 1.001 & .9859
\end{tabular}


The set of $F$ ratios is a pseudo-random sample of size 2500 from $F_{10,26^{\circ}}$ Therefore, the difference between an $F_{10,26}$ point and an $F$ point in Table 3.1 , or the more complete set from which Table 3.1 was drawn, is an estimate of the sampling error inherent in the Monte Carlo simulation. That estimated sampling error was subtracted from each $r a w F_{\hat{A}}$ point (column 5 of Table 3.1) $\hat{\beta}$ to obtain adjusted $F_{\hat{n}}$ points (column 6 of Table 3.1) which we believe are $B$

very nearly equal to the exact $F_{\hat{\beta}}$ points. Both the small- $\sigma$ and large-T approximations were evaluated at each of the 100 adjusted $F_{\hat{n}}$ points.

The four cumulative distributions obtained in this way are plotted in Figure 3.1. The first conclusion to be drawn from this figure is that the small- $\sigma$ approximate $d f$ is nearly identical to the simulated df while the large-T approximate $\mathrm{df}$ is noticeably different from the simulated $\mathrm{df}$. This is true even in the right tails of the distributions which are of most interest for inference. Table 3.2 shows this in more detail. This comparison is strictly true for only the simulated distribution. However, we believe that the use of $F$ as a control variate together with the large number of replications makes it true in general.

These results imply that inference should be based on the small- $\sigma$ approximation. Using the large-T approximation would give a nominal level of confidence which was larger than the true level; that is, the nominal level of significance would be smaller than the true level. Using $F_{10,26}$ as an approximation (i.e. ignoring terms in the approximations beyond the first) would give a nominal level of confidence lower than the true level: that is, the nominal level of significance would be higher than the true leve1. 
Figure 3.1

CUMULATIVE DISTRIBUTIONS
RHO-SQUARED $=.7 \quad T=36 \quad \mathrm{P}=10 \quad \mathrm{~K}=.2857$

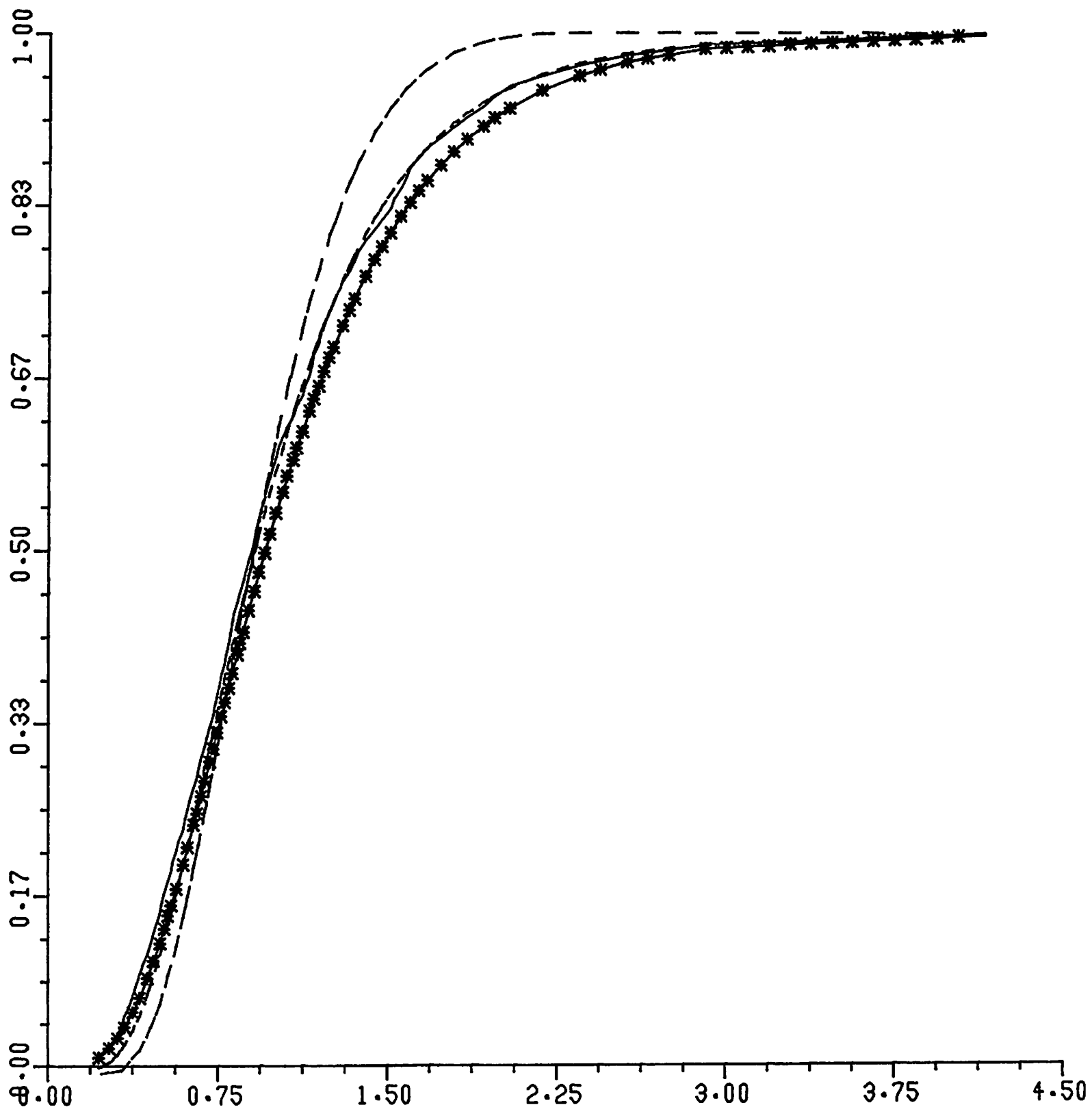

F VALUES

SIMULATED F

APPROXIMATION TO ORDER SIGMA-SQUARED APPROXIMATION TO ORDER $1 / T$

THEORET ICAL $F(10,26)$ 
Although the small- $\sigma$ approximate $d f$ is extremely close to the simulated (and, we believe, the exact) df, it is not an operational basis for inference because it depends upon the unknown parameter $\gamma_{0}=\left(1-\rho^{2}\right) / T$. Fortunately, the range of $\gamma_{0}$ is known because the range of $\rho^{2}$ is known, that is, $0 \leq \rho^{2} \leq 1$ so that $0 \leq \gamma_{0} \leq 1 / T$. At one extreme, as the population goodness-of-fit becomes closer to $1.0, \hat{\beta}$ grows closer to $b$ so the df of $F_{\hat{\beta}}$ gets closer to $F_{p, n}$. For example, the df of $F_{10,26}$ in Figure 3.1 is as far down and right as the approximate (and exact) df can shift. As $\rho^{2}$ grows smaller, making $\gamma_{0}$ larger, the approximate (and exact) df shifts up and left. To illustrate this behavior the small- $\sigma$ approximate df is plotted in Figure 3.2 for values of $\rho^{2}$ of .1 to .9 in increment $s$ of .1 . The furthest left curve corresponds to $\rho^{2}=.1$, the furthest right to $\rho^{2}=.9$. The curves in Figure 3.2 show rather small variation as $\rho^{2}$ changes, given $T$ and $p$. Some more detail from the right tails is shown in Table 3.3. Empirical researchers may have precise prior beliefs about the value of $\rho^{2}$. In the absence of such priors a good rule of thumb seems to be to set $\gamma_{0}$ to the middle of its range, $1 / 2 \mathrm{~T}$; that is, set $\rho^{2}=.5$. The results shown in Figure 3.2 and Table 3.3 suggest that employing such a rule would yield nominal confidence (or significance) levels close to the true levels so long as the nominal confidence level was high. 
Figure 3.2

SMALL-SIGMA CUMULATIVE DISTRIBUTIONS
$T=36 \quad \mathrm{P}=10 \quad \mathrm{~K}=.2857 \quad \mathrm{RHO}-$ SQUARED $=.1 \mathrm{TO} .9$

$己$
$\vdots$
$ت$
0
0
0
0
0
0
0

岁

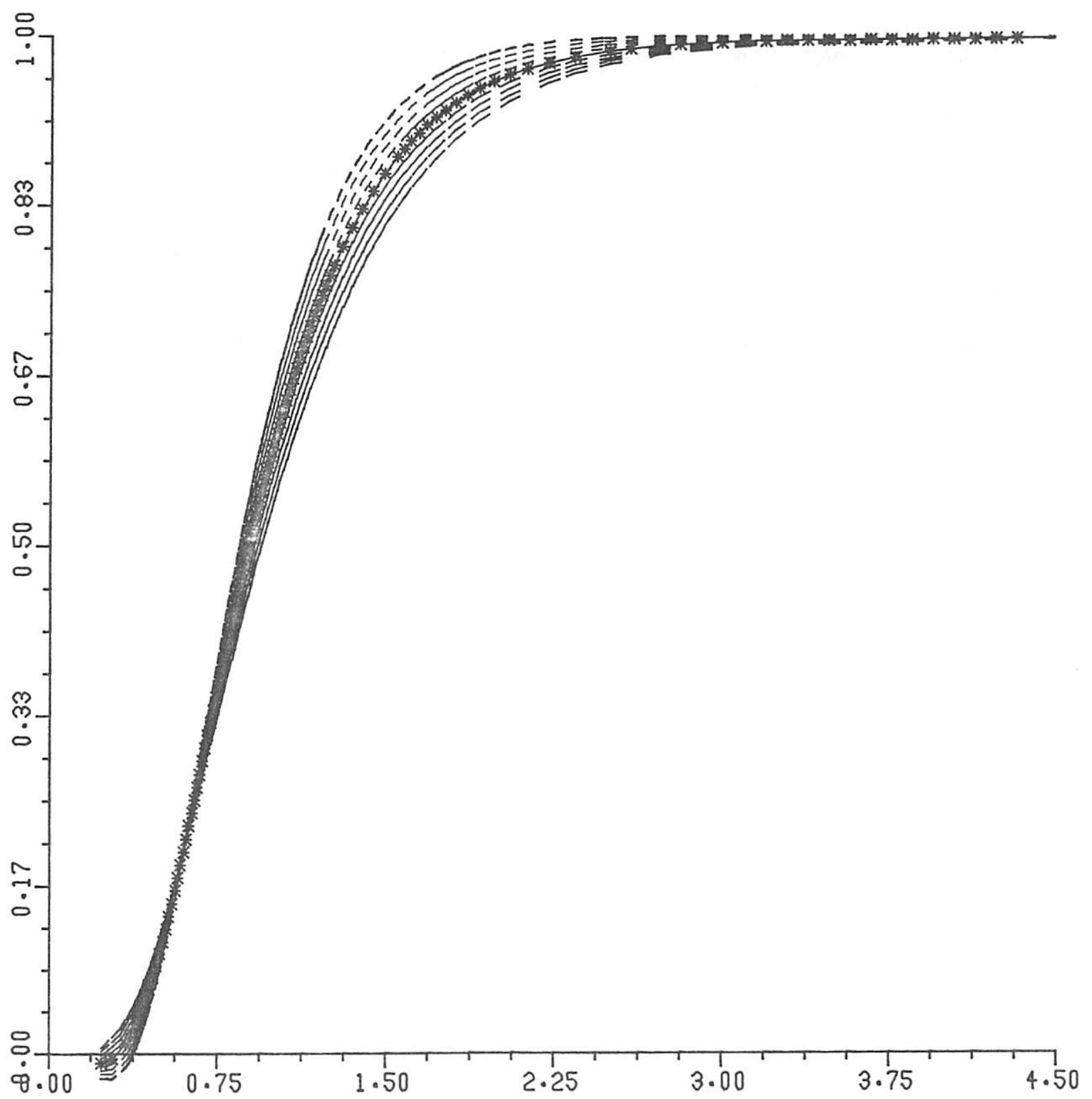

F VALUES

RHO-SQUARED LESS THAN .5 RHO-SQUARED EQUAL TO .5 RHO-SQUARED MORE THAN .5 
TABLE 3.3

Approximate Right Tail Cumulative Probabilities for Various $\rho^{2}$ Values

\begin{tabular}{ccccccccccc} 
& \multicolumn{10}{c}{$\rho^{2}$ Values } \\
\cline { 2 - 10 } F-Values & .1 & .2 & .3 & .4 & .5 & .6 & .7 & .8 & .9 \\
\hline 1.61 & .943 & .932 & .920 & .909 & .897 & .886 & .874 & .863 & .851 \\
1.91 & .980 & .972 & .965 & .957 & .949 & .941 & .933 & .926 & .918 \\
2.04 & .988 & .982 & .975 & .967 & .962 & .956 & .949 & .943 & .936 \\
2.12 & .991 & .986 & .980 & .974 & .968 & .963 & .957 & .951 & .946 \\
2.34 & .997 & .993 & .988 & .984 & .980 & .976 & .972 & .968 & .964 \\
2.71 & 1.000 & .998 & .995 & .993 & .991 & .989 & .987 & .984 & .982
\end{tabular}




\section{DERIVATION OF RESULTS}

In this section we provide proofs of the results in Section $2^{8}$

\subsection{Proof of Theorem 1}

Let us rewrite the model $(2.1)$ as

$(4.1)$

$$
y=x \beta+\sigma v \quad[u=\sigma v]
$$

so that $v$ follows a multivariate normal distribution $N\left(0, I_{T}\right)$ and as $\sigma$ approaches 0 , the disturbance term tends to be small.

Now for the derivation of the result in Theorem 1 we first substitute (4.1) and $(2.2)$ in $(2.4)$ and note that $h=\sigma^{2} \mathrm{kv} ' \mathrm{Mv} / \mathrm{b}^{\prime} \mathrm{Bb}$ is at least of order $\sigma^{2}$. Thus, for sufficiently small $\sigma$ in Kadane's (1971) sense, using (2.2) we can write $(2.3)$ as

(4.2) $\quad \hat{B}=(I+h A)^{-1} b=\left(I-h A+h^{2} A^{2}\right) b+\ldots$

or

$$
\hat{\beta}-\beta=\sigma\left(X^{\prime} X\right)^{-1} X^{\prime} v-h A\left[\beta+\sigma\left(X^{\prime} X\right)^{-1} X^{\prime} v\right]+h^{2} A^{2}\left[\beta+\sigma\left(X^{\prime} X\right)^{-1} X^{\prime} v\right]+\ldots .
$$

where

$$
\text { (4.4) } \quad h=\sigma^{2} k \frac{v^{\prime} M v}{\beta^{\prime} B B}\left[1+2 \sigma^{\beta^{\prime} B\left(X^{\prime} X\right)^{-1} X^{\prime} v}+\sigma^{2} \frac{v^{\prime} X\left(X^{\prime} X\right)^{-1} B\left(X^{\prime} X\right)^{-1} X^{\prime} v}{\beta}\right]^{-1} \text {. }
$$

\section{Define}

$$
s=v^{\prime} M v, z=\left(X^{\prime} X\right)^{-1 / 2} X^{\prime} v=Q^{1 / 2} X^{\prime} v
$$

Notice that $z$ follows a multivariate normal distribution $N\left(0, I_{p}\right)$ and $s$ has a central $x^{2}$-distribution with $n$ degrees of freedom. Further, they are stochastically independent. 
Expanding the expression in square brackets on the right-hand side of (4.4), can express the estimation error in (4.3) up to order $0\left(\sigma^{3}\right)$, as

$(4.6)$

$$
\hat{\beta}-\beta=\sigma e_{1}+\sigma^{2} e_{2}+\sigma^{3} e_{3}
$$

where

$$
\text { (4.7) } \quad e_{1}=Q^{1 / 2} z, e_{2}=-\frac{k s}{\theta} A B, e_{3}=-\frac{k s}{\theta} D . z ; \quad D=\left(A-\frac{2}{\theta} A B B^{\prime} B\right) Q^{1 / 2}
$$

and $\theta=\beta^{\prime} B \beta$ is as given in $(2.7)$.

Thus, up to order $O\left(\sigma^{2}\right)$,

$$
\text { (4.8) } \quad x=\frac{1}{\sigma}(\hat{\beta}-\beta)=e_{7}+\sigma e_{2}+\sigma^{2} e_{3}
$$

and its characteristic function is

$$
\begin{aligned}
\Psi(t) & =E\left[e^{i t^{\prime} x_{j}}\right] \\
& =E_{s} E_{z}\left[e^{i t^{\prime}\left(e_{1}+\sigma e_{2}+\sigma^{2} e_{3}\right)}\right] \\
& =E_{s}\left[e^{-\sigma i \frac{k s}{\theta} t^{\prime} A \beta} E_{z}\left(\left.e^{i \delta^{\prime} z}\right|_{s}\right)\right]
\end{aligned}
$$

where

$$
\text { (4.10) } \quad \delta^{\prime}=t^{\prime}\left[Q^{1 / 2}-\frac{\sigma^{2} k s}{\theta} D\right] .
$$

It is easy to see that

$$
\begin{aligned}
E_{z}\left(\left.e^{i \delta^{\prime} z}\right|_{s}\right) & =e^{-1 / 2 \delta^{\prime} \delta} \\
& \left.=e^{-\frac{1}{2}\left(t^{\prime} Q t-\frac{2 \sigma^{2} k s}{\theta} t\right.} t^{\prime} D Q^{1 / 2} t\right) \\
& =e^{-\frac{1}{2} t^{\prime} Q t}\left[1+\frac{\sigma^{2} k s}{\theta} t Q^{\prime} D Q^{1 / 2} t\right]
\end{aligned}
$$

up to order $0\left(\sigma^{2}\right)$. Substituting $(4.11)$ in $(4.9)$ we get, up to order $0\left(\sigma^{2}\right)$, 


$$
\begin{aligned}
\Psi(t) & =E_{s}\left[\left(1-\sigma i \frac{k s}{\theta} t^{\prime} A \beta-\frac{\sigma^{2}}{2} \frac{k^{2} s^{2}}{\theta^{2}}\left(t^{\prime} A B\right)^{2}\right)\left(1+\sigma^{2} \frac{k s}{\theta} t^{\prime} D Q^{\frac{1}{2}} t\right) e^{-\frac{1}{2} t^{\prime} Q t}\right] \\
& =e^{-\frac{1}{2} t^{\prime} Q t}\left[1-\sigma i \frac{k n}{\theta} t^{\prime} A \beta+\sigma^{2} \frac{k n}{\theta^{2}} t^{\prime}\left\{\theta D Q^{\frac{1}{2}}-\frac{1}{2}(n+2) k A B B^{\prime} A\right\} t\right] .
\end{aligned}
$$

Next, using the Inversion Theorem, the multivariate density function of $x$ to order $0\left(\sigma^{2}\right)$ is

$$
\begin{aligned}
g_{0}(x) & =\frac{1}{(2 \pi)^{p}} \int_{-\infty}^{\infty} \cdots \int_{-\infty}^{\infty} e^{-i t^{\prime} x} \Psi(t) d t \\
& =g(x)\left[1-\sigma^{\frac{k n}{\theta}} x^{\prime} Q^{-1} A B+\sigma^{2} \frac{k n}{\theta^{2}}\left\{\theta \operatorname{tr} A-2 \beta^{\prime} B A B\right.\right. \\
& \left.\left.-\frac{1}{2}(n+2) k \beta^{\prime} A Q^{-1} A B-x^{\prime}\left(\theta Q^{-1} A-2 Q^{-1} A B B^{\prime} B-\frac{1}{2}(n+2) k Q^{-1} A B \beta^{\prime} A Q^{-1}\right) x\right\}\right]
\end{aligned}
$$

where we have utilized the following results:

$$
\frac{1}{(2 \pi)^{P}} \int_{-\infty}^{\infty} \ldots \int_{-\infty}^{\infty} e^{-i t^{\prime}: x-\frac{1}{2} t^{\prime} Q t} d t=g(x)=\frac{e^{-\frac{1}{2} x^{\prime} Q^{-1} x}}{(2 \pi)^{\frac{P}{2}}|Q|^{\frac{1}{2}}}
$$

$(4.14)$

$$
\begin{aligned}
& \frac{1}{(2 \pi)^{P}} \int_{-\infty}^{\infty} \ldots \int_{-\infty}^{\prime} \delta_{0}^{\prime} t e^{-i t^{\prime} x-\frac{1}{2} t^{\prime} Q t} d t=-i \delta_{0}^{\prime} Q^{-1} x g(x) \\
& \frac{i}{(2 \pi)^{P}} \int_{-\infty}^{\infty} \ldots \int_{-\infty}^{\infty} t^{\prime} D_{0} t e^{-i . t^{\prime} x-\frac{1}{2} t^{\prime} Q t} d t=\left[t r D_{0} Q^{-1}-x^{\prime} Q^{-1} D_{0} Q^{-1} x\right] g(x)
\end{aligned}
$$

for any fixed vector $\delta_{0}$ and fixed matrix $D_{0}$.

Finally, noting that for any fixed vector $\delta_{1}$ and the matrix $D_{1}$

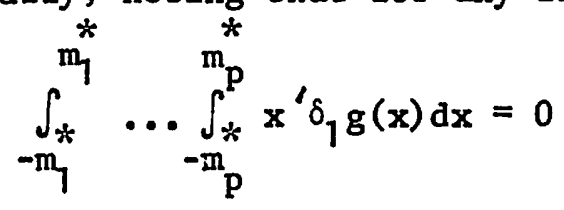

and

$$
\int_{-m_{1}}^{m_{1}^{*}} \ldots \int_{-m_{p}^{*}}^{m_{p}^{*}} x^{\prime} D_{1} x g(x) d x=\left(G\left(m^{*}\right)-G\left(-m^{*}\right)\right)\left[\operatorname{tr} D_{1} Q-\operatorname{tr} Q^{\frac{1}{2}} D_{1} Q^{\frac{1}{2}} \Gamma\right]
$$


we get $P\left[-m_{0}<x<m_{0}\right]$ as stated in Theorem $1\left(m_{*}=Q^{-\frac{1}{2}} m_{0}\right)$.

\subsection{Proof of Theorem 2}

From (4.7) and (4.8)

(4.17) $\quad \tau^{\prime} x=\frac{1}{\sigma} \tau^{\prime}(\hat{\beta}-\beta)=\delta_{2}^{\prime} z-\sigma \frac{k s}{\theta} z^{\prime} A \beta$

where

(4.18) $\delta_{2}^{\prime}=\tau^{\prime} Q^{\frac{1}{2}}\left[I_{p}-\sigma^{2} \frac{\mathrm{ks}}{\theta} Q^{-\frac{1}{2}} \mathrm{AQ}^{\frac{1}{2}}\left(I_{\mathrm{p}}-\frac{2}{\theta} Q^{-\frac{1}{2}} \mathrm{BB}^{\prime} \mathrm{BQ}^{\frac{1}{2}}\right)\right]$.

Now the characteristic function of $z^{\prime} x$ is

(4.19)

$$
\begin{aligned}
\Psi(t) & =E\left[e^{i t\left(z^{\prime} x\right)}\right] \\
& =E_{s} E_{z}\left[e^{i t\left(\delta_{2}^{\prime} z-\sigma \frac{k z^{\prime} A B}{\theta}\right)}||_{s}\right. \\
& =E_{s}\left[e^{-i t\left(\sigma \frac{k s z^{\prime} A B}{\theta}\right)} E_{z}\left\{e^{i t\left(\delta_{2}^{\prime} z\right)}||_{s}\right]\right.
\end{aligned}
$$

It is easy to see that

(4.20) $\quad E_{z}\left\{\left.e^{i t\left(\delta_{2}^{\prime} z\right)}\right|_{s}\right\}=e^{-\frac{1}{2} \delta_{2}^{\prime} \delta_{2} t^{2}}$.

But, up to order $0\left(\sigma^{2}\right)$

(4.21) $\quad \delta_{2}^{\prime} \delta_{2}=z^{\prime} \mathrm{Q} z-2 \sigma^{2} \frac{\mathrm{ks}}{\theta}\left\{z^{\prime} \mathrm{AQ} z-\frac{2}{\theta} z^{\prime} \mathrm{ABB}{ }^{\prime} \mathrm{BQ} z\right\}$.

Thus putting $(4.21)$ in $(4.20)$, and then substituting $(4.20)$ in $(4.19)$ along with

$$
e^{-i t\left(\frac{\sigma k s z^{\prime} A B}{\theta}\right)}=1-\sigma i t \frac{k s z^{\prime} A B}{\theta}-\sigma^{2} \frac{t^{2}}{2}\left(\frac{k s^{\prime} A B}{\theta}\right)^{2}
$$

we find, up to order $0\left(\sigma^{2}\right)$ 


$$
\begin{aligned}
& (4.23) \quad \Psi(t)=e^{-\frac{t^{2} q^{2}}{2}} E_{s}\left[1-\sigma i t \frac{k s z^{2} A \beta}{\theta}-\sigma^{2} \frac{t^{2} k^{2} s^{2}\left(z^{\prime} A \beta\right)^{2}}{2 \theta^{2}}\right. \\
& \left.+\sigma^{2} \frac{t^{2} k s}{\theta}\left\{z^{\prime} A Q z-\frac{2}{\theta} \tau^{\prime} A B \beta B^{\prime} \mathrm{BQ} z\right\}\right] \\
& =e^{-\frac{t^{2} q^{2}}{2}}\left[1-\sigma \frac{i t k n z ' A \beta}{\theta}-\sigma^{2} \frac{t^{2} k n}{\theta}\left[\frac{k(n+2)}{2 \theta}\left(\tau^{\prime} A \beta\right)^{2}\right.\right. \\
& \left.\left.+\frac{2}{\theta} \tau^{\prime} \mathrm{ABB}{ }^{\prime} \mathrm{BQ} z-\tau^{\prime} \mathrm{AQ} z\right\}\right]
\end{aligned}
$$

where $\mathrm{q}^{2}=\iota^{\prime} \mathrm{Q} \imath$ as given in (2.7).

Next, using the Inversion Theorem, the density function of $2^{\prime} x$ to order $0\left(\sigma^{2}\right)$ is

$$
\begin{aligned}
& \left.g_{0}\left(\tau^{\prime} x\right)=\frac{1}{2 \pi} \int_{-\infty}^{\infty} e^{-i t \tau^{\prime} x_{\Psi}} \Psi t\right) d t \\
& =\frac{1}{q} g\left(\frac{z^{\prime} x}{q}\right)\left[1-\sigma \frac{k n z^{\prime} A B}{\theta} \frac{z^{\prime} x}{q^{2}}+\sigma^{2} \frac{k n}{\theta q^{2}}\left\{\frac{k(n+2)}{2 \theta}\left(z^{\prime} A \beta\right)^{2}\right.\right. \\
& \left.\left.+\frac{2}{\theta} \tau^{\prime} \mathrm{ABB} \mathrm{B}^{\prime} \mathrm{BQ} z-\tau^{\prime} \mathrm{AQ} z\right\}\left(\frac{\left(2^{\prime} \mathrm{x}\right)^{2}}{\mathrm{q}^{2}}-1\right)\right]
\end{aligned}
$$

where we have utilized the following results:

$$
\begin{aligned}
& \frac{1}{2 \pi} \int_{-\infty}^{\infty} e^{-\frac{t^{2} q^{2}}{2}-i t \tau^{\prime} x} d t=\frac{1}{q} g\left(\frac{z^{\prime} x}{q}\right) \\
& \frac{1}{2 \pi} \int_{-\infty}^{\infty} i t e^{-\frac{t^{2} q^{2}}{2}-i t \tau^{\prime} x} d t=\frac{z^{\prime} x}{q^{3}} g\left(\frac{z^{\prime} x}{q}\right) \\
& \frac{1}{2 \pi} \int_{-\infty}^{\infty}(i t)^{2} e^{-\frac{t^{2} q^{2}}{2}-i t z^{\prime} x} d t=\left(\frac{\left(z^{\prime} x\right)^{2}}{q^{2}}-1\right) \frac{1}{q^{3}} g\left(\frac{z^{\prime} x}{q}\right) .
\end{aligned}
$$

Finally, noting that the distribution function $P\left[\tau^{\prime} x \leq m\right]$ $=\int_{-\infty}^{\mathfrak{m}} g_{0}\left(z^{\prime} x\right) d z^{\prime} x$, we get the result in Theorem 2 . 
26

4.3 Proof of Theorem 3

Let us write by using $(4.6)$ and $(4.7)$

$$
(\hat{\beta}-\beta){ }^{\prime} W(\hat{\beta}-\beta)=\sigma^{2} e_{1}^{\prime} W e_{1}+2 \sigma^{3} e_{1}^{\prime} W e_{2}+\sigma^{4}\left(e_{2}^{\prime} W e_{2}+2 e_{1}^{\prime} W e_{3}\right),
$$

up to order $0\left(\sigma^{4}\right)$, where $W$ is any positive definite matrix and

$$
e_{1}^{\prime} W_{1}=z^{\prime} Q^{\frac{1}{2}} W^{\frac{1}{2}} z, e_{1}^{\prime} W_{2}=-\frac{k s}{\theta} \beta^{\prime} A^{\frac{1}{2}} z Q_{z}
$$

$(4.27)$

$$
e_{2}^{\prime} W e_{2}=\frac{k^{2} s^{2}}{\theta^{2}} \beta^{\prime} A W A \beta, e_{1}^{\prime} W e_{3}=-\frac{k s}{\theta} z^{\prime} Q^{\frac{1}{2}} W^{\frac{1}{2}}\left(I_{p}-\frac{2}{\theta} Q^{-\frac{1}{2}} \beta B^{\prime} B^{\frac{1}{2}}\right) z .
$$

Substituting $(4.27)$ in $(4.26)$ and dividing both sides of $(4.26)$ by $\sigma^{2} \mathrm{~s}$ we can write the improved $F$ ratio in $(2.26)$ as

(4.28) $\quad \mathrm{P}_{\mathrm{n}} \mathrm{F}_{\hat{\beta}}=\mathrm{F}_{\hat{\beta}}^{0}=\frac{(\hat{\beta}-\beta)^{\prime} W(\hat{\beta}-\beta)}{\sigma^{2} s}=z^{\prime} P_{1} z+\sigma \delta_{3}^{\prime} z+\sigma^{2} s \beta^{\prime} P_{2} \beta$

where

$$
P_{1}=H_{1}+\sigma^{2} H_{2} ; H_{1}=\frac{Q^{\frac{1}{2}} \frac{1}{2} Q^{\frac{1}{2}}}{s}, H_{2}=-\frac{2 k}{\theta} Q^{\frac{1}{2}} \operatorname{WAQ}^{\frac{1}{2}}\left[I_{p}-\frac{2}{\theta} Q^{-\frac{1}{2}} \beta \beta^{\prime} Q^{\frac{1}{2}}\right]
$$

$$
P_{2}=\frac{k^{2}}{\theta^{2}} A W A, \delta_{3}^{\prime}=-\frac{2 k}{\theta} \beta^{\prime} A^{\frac{1}{2}} \text {. }
$$

Now the characteristic function of $F_{\hat{\beta}}^{0}$ is

$$
\begin{aligned}
\phi(t)=E e^{i t F_{\hat{\beta}}^{0}} & =E_{s} E_{z}\left[\left.e^{i t\left(z^{\prime} P_{1} z+\sigma \delta_{3}^{\prime} z+\sigma^{2} s \beta^{\prime} P_{2}^{\beta}\right.}\right|_{s}\right] \\
& =E_{s}\left[e^{i t \sigma^{2} s \beta^{\prime} P_{2} \beta} E_{z}\left\{\left.e^{i t\left(z^{\prime} P_{1} z+\sigma \delta_{3}^{\prime} z\right)}\right|_{s}\right\}\right] .
\end{aligned}
$$

Using (4.29), it is easy to see that up to order $O\left(\sigma^{2}\right)$ 
$(4.31)$

$$
\begin{aligned}
E_{z}\left\{\left.e^{i t\left(z^{\prime} P_{1} z+\sigma \delta_{3}^{\prime} z\right)}\right|_{s}\right\} & =\left|I_{p}-2 i t P_{1}\right|^{-\frac{1}{2}} e^{-\frac{t^{2}}{2} \sigma^{2} \delta_{3}^{\prime}\left(I_{p}-2 i t P_{1}\right)^{-1} \delta_{3}} \\
& =\left|J_{1}\right|^{-\frac{1}{2}}\left[1+i t \sigma^{2} t r J_{1}^{-1} H_{2}-\frac{t^{2}}{2} \sigma^{2} \delta_{3}^{\prime} J_{1}^{-1} \delta_{3}\right],
\end{aligned}
$$

where

(4.32) $J_{1}=I_{p}-2 i t H_{1}$,

and we use the result that for any square matrix $A,|I-A|^{-\frac{1}{2}} \approx 1+\frac{1}{2} \operatorname{tr} A$. Also, up to order $0\left(\sigma^{2}\right)$

(4.33) $e^{i t \sigma^{2} s \beta^{\prime} P_{2} \beta}=1+i t \sigma^{2} s \beta{ }^{\prime} P_{2} \beta$.

Thus, substituting $(4.31)$ and $(4.32)$ in $(4.30)$ we get, up to order $0\left(\sigma^{2}\right)$

$$
\begin{gathered}
\phi(t)=E_{s}\left[\left(1+i t \sigma^{2} s \beta^{\prime} P_{2} \beta\right)\left|J_{1}\right|^{-\frac{1}{2}}\left(1+i t \sigma^{2} \operatorname{tr} J_{1}^{-1} H_{2}-\frac{t^{2}}{2} \sigma^{2} \delta_{3}^{\prime} J_{1}^{-1} \delta_{3}\right)\right] \\
=E_{s}\left(\left|J_{1}\right|^{-\frac{1}{2}}\right)+i t \sigma^{2} \operatorname{tr~}_{2} E_{s}\left(\left|J_{1}\right|^{-\frac{1}{2}} J_{1}^{-1}\right)-\frac{t^{2}}{2} \sigma^{2} \delta_{3}^{\prime} E_{s}\left(\left|J_{1}\right|^{-\frac{1}{2}} J_{1}^{-1}\right) \delta_{3} \\
+i t \sigma^{2} \beta^{\prime} P_{2} \beta E_{s}\left(s\left|J_{1}\right|^{-\frac{1}{2}}\right)
\end{gathered}
$$

Considering $W=X^{\prime} X$ in $(4.28)$, we note that $E_{s}\left|J_{1}\right|^{-\frac{1}{2}}=E_{s}\left(1-\frac{2 i t}{s}\right)^{-\frac{p}{2}}$ $=\phi\left(F_{p, n}^{O}, t\right)$; that is the characteristic function of a central $\frac{p}{n} F=F^{0}$ ratio with the $p$ and $n$ degrees of freedom, see Phillips (1982, p. 262). Similarly, it can easily be verified that $E_{s}\left(\left|J_{1}\right|^{-\frac{1}{2}} J_{1}^{-1}\right)=E_{s}\left[\left(1-\frac{2 i t}{s}\right)^{-\frac{(p+2)}{2}}\right] I_{p}$ where $E_{s}\left(1-\frac{2 i t}{s}\right)^{-\frac{(p+2)}{2}}=\phi\left(F_{p+2, n}^{o}, t\right)$. Also, $E_{s} \frac{s}{n}\left|J_{1}\right|^{-\frac{1}{2}}=E_{s} \frac{s}{n}\left(1-\frac{2 i t}{s}\right)^{-\frac{p}{2}}$ $=\phi\left(\mathrm{F}_{\mathrm{p}, \mathrm{n}+2}^{\mathrm{o}}, \mathrm{t}\right)$. Using these results in $(4.34)$ we can write 
$(4.35)$

$$
\begin{aligned}
\phi(t)=\phi\left(F_{p, n}^{o}, t\right) & +i t \sigma^{2}\left(t r H_{2}\right) \phi\left(F_{p+2, n}^{o}, t\right)-\left(\frac{t^{2}}{2} \sigma^{2} \delta_{3}^{\prime} \delta_{3} \phi\left(F_{p+2, n}^{o}, t\right)\right. \\
& +i t \sigma^{2} n\left(\beta^{\prime} P_{2} \beta\right) \phi\left(F_{p, n+2}^{o}, t\right) .
\end{aligned}
$$

Now, using the Inversion Theorem, the density function of the $F$ ratio in (4.28), with $W=X^{\prime} X$, can be written after simplification as

$$
\begin{aligned}
g_{0}\left(F_{\hat{\beta}}^{0}\right)=g\left(F_{p, n}^{0}\right) & +\eta\left[g\left(F_{p, n+4}^{o}\right)-g\left(F_{p-2, n+6}^{o}\right)\right]+\eta_{1,1}\left[g\left(F_{p+2, n+2}^{o}\right)\right. \\
& \left.-g\left(F_{p, n+4}^{0}\right)\right]+\frac{2 \eta_{1,2}}{(n+2)}\left[\left(\frac{n}{2}+1\right) g\left(F_{p+2, n+4}^{o}\right)+\left(\frac{n}{2}+3\right) g\left(F_{p-2, n+8}^{o}\right)\right. \\
& \left.-2\left(\frac{n}{2}+2\right) g\left(F_{p, n+6}^{o}\right)\right]
\end{aligned}
$$

where $\eta, \eta_{1,1}$ and $\eta_{1,2}$ are as given in (2.28). In obtaining the result in (4.36) we used the following results (for any positive scalar $F^{\circ}$ ):

$$
\begin{aligned}
& \frac{1}{2 \pi} \int_{-\infty}^{\infty} e^{-i t F^{0}} \phi\left(F_{p, n}^{0}, t\right) d t=g\left(F_{p, n}^{0}\right)=\frac{\Gamma\left(\frac{p+n}{2}+1\right)}{\Gamma\left(\frac{p}{2}+1\right) \Gamma\left(\frac{n}{2}\right)} \frac{F^{0(p+2) / 2-1}}{\left(1+F^{0}\right)^{(p+2+n) / 2}} \\
& \frac{1}{2 \pi} \int_{-\infty}^{\infty} i t e^{-i t F^{0}} \phi\left(F_{p, n+2}^{0}, t\right) d t=\frac{(n+2)(n+4)}{2(p+n+2)}\left[g\left(F_{p, n+4}^{0}\right)-g\left(F_{p-2, n+6}^{0}\right)\right]
\end{aligned}
$$

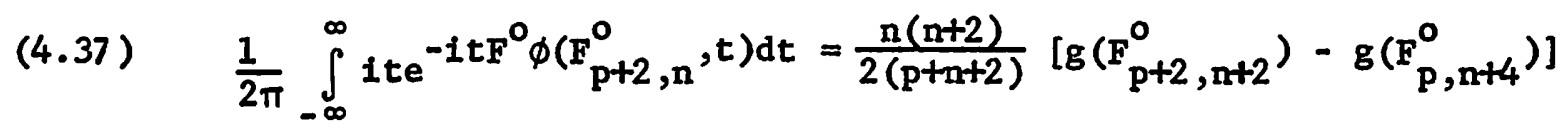

$$
\begin{aligned}
\frac{1}{2 \pi} \int_{-\infty}^{\infty} t^{2} e^{-i t F^{0}} \phi\left(F_{p+2, n}^{0}, t\right) d t= & \frac{n(n+2)}{(p+n+2)(p+n+4)}\left[( \frac { n } { 2 } + 2 ) ( \frac { n } { 2 } + 1 ) \left\{g\left(F_{p+2, n+4}^{0}\right)\right.\right. \\
& \left.-g\left(F_{p, n+6}^{0}\right)\right\}-\left(\frac{n}{2}+3\right)\left(\frac{n}{2}+2\right)\left\{g\left(F_{p, n+6}^{0}\right)\right. \\
& \left.\left.-g\left(F_{p-2, n+8}^{0}\right)\right\}\right]
\end{aligned}
$$


Finally, noting that the distribution function of $F_{\hat{\beta}}^{0}$ is $\mathrm{P}\left[\mathrm{F}_{\hat{\beta}}^{0} \leq \mathrm{m}\right]=\int_{0}^{\mathrm{m}} \mathrm{g}_{0}\left(\mathrm{~F}_{\hat{\beta}}^{0}\right) \mathrm{dF} \mathrm{\beta}_{\hat{\beta}}^{0}$, and observing that $\mathrm{P}\left(n \mathrm{~F}_{\hat{\beta}}^{0} \leq \mathrm{m}\right)=\mathrm{P}\left(\mathrm{pF}_{\hat{\beta}} \leq \mathrm{m}\right)$ we get the result stated in Theorem 3 . 


\section{FOOTNOTES}

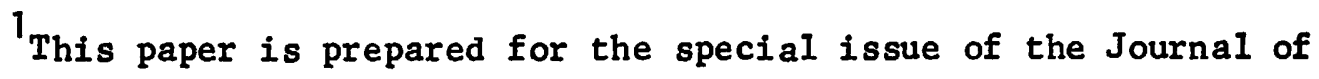
Econometrics on Shrinkage and Pre-test Estimators. The research supports to A. UIlah from the NSERC and Air Force Office of Scientific Research at the Center for Multivariate Analysis, University of Pittsburgh, and to R. Carter from SSHRCC, are gratefully acknowledged: The authors are thankful to D.E.A. Giles for helpful discussions and T. Peters for research assistance.

2 The multivariate density function of $x=(\hat{\beta}-\beta) / \sigma$ is given in (4.13). Using this, one can easily verify that $E \hat{\beta}$ and $E(\hat{\beta}-\beta) X^{\prime} X(\hat{\beta}-\beta)$ are identical with the corresponding results in Ullah et al (1983) for the normal case. Another point to be noted is that when $A$ in (2.3) is not a symmetric matrix, the results stated in Theorems 1 to 3 remain the same with $B$ 'A replaced by $B^{\prime} A^{\prime}$. Since in almost all the shrinkage estimators known in the literature A is a symmetric matrix we have presented here results for this case only.

${ }^{3}$ Given the distribution of $2^{\prime} x$ in $(2.10)$ the density function of $z^{\prime} x$ can be obtained (also see (4.24)). Using this density one can also verify the results indicated in footnote 2 .

4he result in (2.23) compares with that in UIlah (1982).

5 The result in this section easily extends to the case of testing linear restrictions $R \beta=r$, where $R$ is an $m \times p$ matrix and $r$ is an $m \times 1$ vector of known constants. This is because we can write $R \beta=r$ as $R \beta=R \beta_{0}$ or $R\left(\beta-\beta_{0}\right)=0$, where $\beta_{0}$ is a fixed vector, and hence the $F$ statistic as $\left(b-\beta_{0}\right)^{\prime} W\left(b-\beta_{0}\right) / \hat{u}^{\prime} \hat{u}$, where $W=R^{\prime}\left[R\left(X^{\prime} X\right)^{-1} R^{\prime}\right]^{-1} R$. In fact, the proof in Section 4.3 has been 
outlined for any positive definite matrix $W$. We have considered here $R=I_{p}\left(W=X^{\prime} X\right)$ for the sake of simplicity in exposition.

Gote that $\mathrm{pF}_{\hat{\hat{B}}}=(\hat{\beta}-\beta)^{\prime}[\mathrm{Asy} \cdot \mathrm{V}(\hat{\beta})]^{-1}(\hat{\beta}-\beta)$ is the well-known Wald (1943) test statistic, where Asy.V( $\hat{\beta})=\sigma^{2}\left(X^{\prime} X\right)^{-1}$ is the asymptotic variance of $\hat{\beta}$ and it is to be evaluated at the unrestricted estimator of $\sigma^{2}$. We have taken $\hat{\sigma}^{2}=\hat{u}^{\prime} \hat{u} / \mathrm{n}$ where $\hat{\mathrm{u}}=\mathrm{y}-\mathrm{Xb}$. An alternative to this, that is $\tilde{\sigma}^{2}=\tilde{u}{ }^{\prime} \tilde{u} / \mathrm{n}$, where $\tilde{u}=y-X \hat{\beta}$, was also considered. In this case the result in (2.27) of Theorem 3 remained the same with $[\operatorname{tr} A-2 \beta B A \beta / \theta]$ on the right-hand side of $\eta_{1,1}\left(\right.$ see $(2.28)$ ) replaced by $\left[\operatorname{tr} A-2 \beta^{\prime} B A \beta / \theta+k \beta^{\prime} A X^{\prime} X A \beta / 2 \theta\right]$. Further, the numerical findings in Section 3 would be changed only slightly by using $\tilde{\sigma}^{2}$, or the approximate $V(\hat{\beta})$ given in UIlah and Ullah (1978) instead of Asy.V $(\hat{\beta})$.

${ }^{7}$ A less interesting case of the distribution of the statistic $(\hat{\beta}-\beta)^{\prime} X X^{\prime} X(\hat{\beta}-\beta) / \sigma^{2}$ was also examined and it was found that while the small- $\sigma$ expansion of this statistic was in terms of the central $\chi^{2}$ distribution and hence different with (2.29), the large-T expansion was the same as in (2.30). This implies that the replacement of $\sigma^{2}$ by $\hat{u}^{\prime} \hat{u} / n$ did not change the large-T expansion up to the order of approximation considered.

${ }^{8}$ We essentially derive Edgeworth-type expansions. For details about these expansions, see Sargan (1976). 


\section{REFERENCES}

Baranchik, A. J., 1964, "Multiple Regression and Estimation of the Mean of a Multivariate Normal Distribution," Technical Report 51 (Stanford Department of Statistics).

Carter, R. A. I., 1981 , "Improved Stein-Rule Estimator for Regression Problems," Journal of Econometrics 17, 113-123.

Danie1, C. and F. Wood, 1971, Fitting Equations to Data (Wiley: New York)

Hoer 1, A. E., R. W. Kennard and K. F. Baldwin, 1975, "Ridge Regression: Some Simulations," Communications in Statistics, 108-115.

James, W. and C. Stein, 1961, "Estimation with Quadratic Loss," Proceedings of Fourth Berkeley Symposium in Mathematical Statistics and Probability $1,1020-1034$

Judge, G. G., and M. E. Bock, 1978, The Statistical Implications of Pretest and Stein-Rule Estimators in Econometrics (North-Holland: New York).

Judge, G. G., W. E. Griffiths, R. C. Hill and T. C. Lee, 1980, The Theory and Practive of Econometrics (Wiley: New York).

Kadane, J. B., 1971, "Comparison of k-class Estimators When the Disturbances are Smal1," Econometrica (Sept.), 723-737.

Lawless, J.F. and P. Wang, 1976, "A Simulation Study of Ridge and Other Regression Estimators," Communications in Statistics, Ser. A, 307-323.

Nagar, A. L., 1959, "The Bias and Moment Matrix of the General k-class Estimators of Parameters in Simultaneous Equations," Econometrica (Oct.), $575-595$

Phillips, P. C. B., 1982, "The True Characteristic Function of the F Distribution," Biometrika 69, $261-264$. 
Rao, C. R., 1973, Linear Statistical Inference and its Applications (Wiley: New York).

Sargan, J. D., 1976, "Econometric Estimators and the Edgeworth Approximation," Econometrica $44,421-428$.

Srivastava, V. K., T. D. Dwivedi, M. Belinsky and R. Tiwari, 1980, "A Numerical Comparison of Exact, Large-Sample and Sma11-Disturbance Approximations of Properties of k-class Estimators," International Economic Review (Feb.), 249-252.

Stein, C., 1966 "An Approach to the Recovery of Inter-Block Information on Balanced Incomplete Block Designs" in F. N. David (ed.), Research Papers in Statistics (Wiley: New York), 351-366.

U1lah, A., 1974, "On the Sampling Distribution of Improved Estimators for Coefficients in Linear Regression," Journal of Econometrics, 143-150. U11ah, A., 1980, "The Exact, Large-Sample and Small-Disturbance Conditions of Dominance of Biased Estimators in Linear Models," Economics Letters, $339-344$.

U1Iah, A., 1982, "The Approximate Distribution Function of the Stein-Rule Estimator," Economics Letters, 305-308.

UIlah, A., and S. UIlah, 1978, "Double k-class Estimators of Coefficients in Linear Regression," Econometrica (May).

Ullah, A., V. K. Srivastava and R. Chandra, 1983, "Properties of Shrinkage Estimators in Linear Regression When Disturbances Are Not Normal," Journal of Econometrics (Apr.), 389-402.

Vinod, H. D. and A. UIlah, 1981, Recent Advances in Regression Methods (Marcel Dekkar: New York).

Wald, A., 1943, "Iests of Hypotheses Concerning Several Parameters When the Number of Observations is Large," Transactions of the American Mathematical Society $54,426-482$. 